\title{
Epidemiology of Prostate and Testicular Cancer
}

\author{
Pauline Filippou, MD ${ }^{1}$ James E. Ferguson III, MD, $\mathrm{PhD}^{1} \quad$ Matthew E. Nielsen, MD, MS ${ }^{1}$ \\ ${ }^{1}$ Department of Urology, University of North Carolina at Chapel Hill, \\ Chapel Hill, North Carolina \\ Address for correspondence Pauline Filippou, MD, Department of \\ Urology, University of North Carolina at Chapel Hill, 2107 Physicians \\ Office Building, Campus Box 7235, Chapel Hill, NC 27599 \\ Semin Intervent Radiol 2016;33:182-185 \\ (e-mail: Pauline.Filippou@unchealth.unc.edu).
}
Abstract
Keywords
- epidemiology
- men's health
- prostate cancer
- testicular cancer
- interventional radiology

Prostate and testicular cancers account for a large percentage of cancer morbidity in men in the United States and worldwide due to high prevalence rates that continue to grow. Patterns of incidence and mortality vary greatly in both cancers among men of different age groups, ethnicities, and geographic locations. This article summarizes the incidence, prognosis, and risk factors of both prostate and testicular cancers, globally and in the United States.
Objectives: Upon completion of this article, the reader will be able to identify the incidence and mortality trends of prostate and testicular cancers in the United States and globally, as well as discuss the risk factors for prostate and testicular cancers.

Accreditation: This activity has been planned and implemented in accordance with the Essential Areas and Policies of the Accreditation Council for Continuing Medical Education (ACCME) through the joint providership of Tufts University School of Medicine (TUSM) and Thieme Medical Publishers, New York. TUSM is accredited by the ACCME to provide continuing medical education for physicians.

Credit: Tufts University School of Medicine designates this journal-based CME activity for a maximum of 1 AMA PRA Category 1 Credit $^{\mathrm{TM}}$. Physicians should claim only the credit commensurate with the extent of their participation in the activity.

In the United States and worldwide, the prevalence of prostate and testicular cancers has been steadily increasing over the past several decades. While 1 in 7 men will be diagnosed with prostate cancer at some point in their life, only 1 in 263 men will be diagnosed with testicular cancer. ${ }^{1}$ However, testicular cancer is the most common cancer diagnosed in men aged 15 to 35 . This article will review the incidence, prognosis, and risk factors of both prostate and testicular cancers, and specifically quantify the morbidity of both cancers in the United States today.

\section{Prostate Cancer Incidence}

The incidence of prostate cancer on the global scale varies widely and is significantly affected by implementation of health care resources and epidemiologic resources. Worldwide, prostate cancer is the fourth most common cancer (1.1 million new cases in 2012, accounting for $15 \%$ of cancer diagnosed), with $70 \%$ of diagnoses occurring in developed regions. ${ }^{2}$ Incidence rates vary more than 25 -fold worldwide, with the highest incidence rates seen in the developed regions of Australia/New Zealand, Western and Northern Europe, and Northern America, where the practice of cancer screening with prostate-specific antigen (PSA) and subsequent pathologic diagnosis are common (-Fig. 1). ${ }^{2}$

In the United States, prostate cancer is the most common noncutaneous malignancy diagnosed in men. According to data from the United States National Cancer Institute's Surveillance Epidemiology and End Results (SEER) database, in 2015 a total of 220,800 men were diagnosed with prostate cancer in the United States, with an age-adjusted incidence rate between 2008 and 2012 of 137.9 per 100,000 men per year. The reported incidence of prostate cancer over the past 30 years in the United States has been highly sensitive to screening, as well as other health care phenomena that result in histologic evaluation of prostate tissue (i.e., transurethral resection of the prostate [TURP] for benign prostatic hyperplasia). Starting in 1980, the incidence of prostate cancer steadily
Issue Theme Men's Health; Guest Editor, Charles Burke, MD
Copyright @ 2016 by Thieme Medical Publishers, Inc., 333 Seventh Avenue, New York, NY 10001, USA. Tel: +1(212) 584-4662.
DOI http://dx.doi.org/ 10.1055/s-0036-1586146. ISSN 0739-9529. 


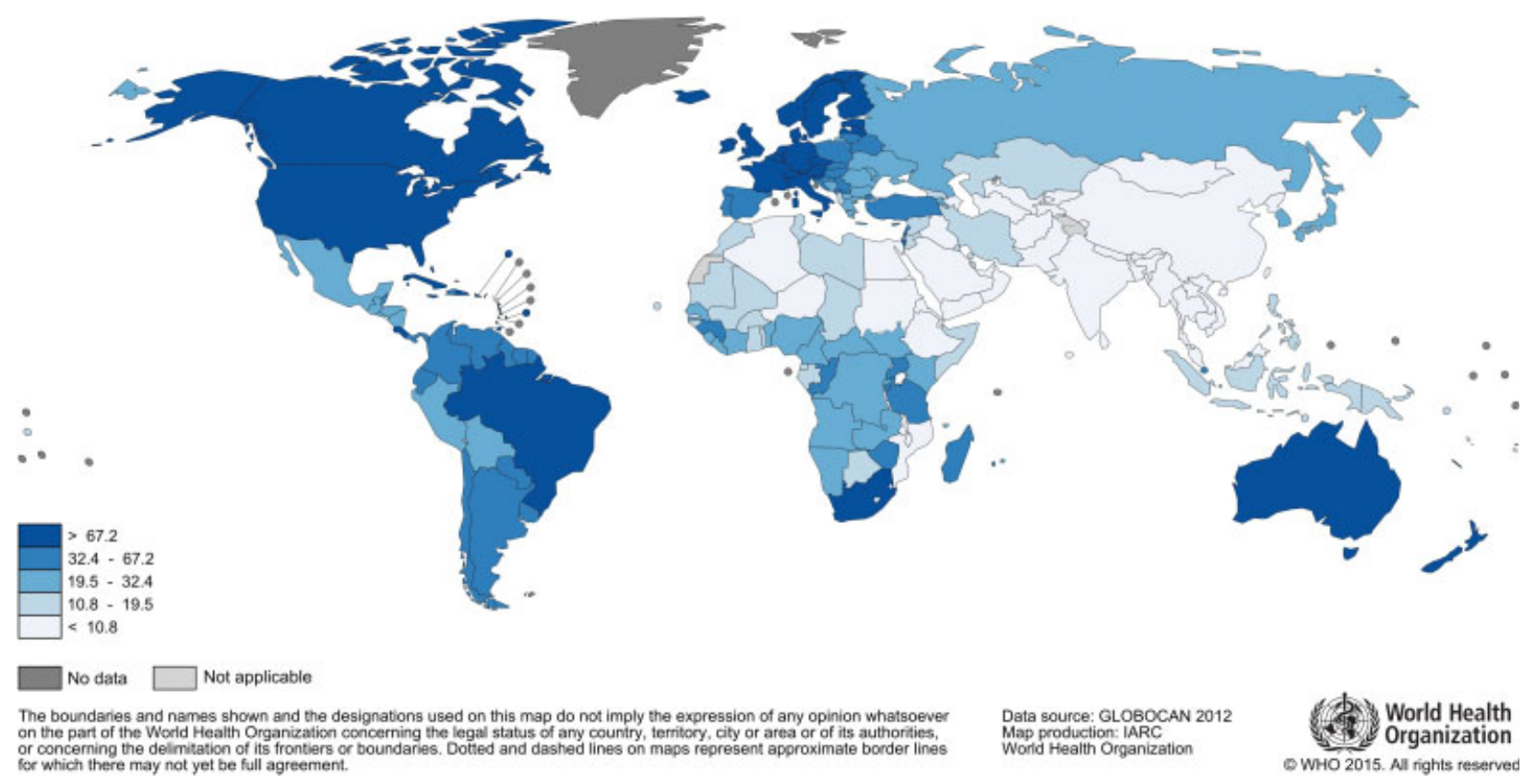

Fig. 1 Estimated prostate cancer incidence worldwide in 2012. Source: GLOBOCAN 2012 http://globocan.iarc.fr/Pages/fact_sheets_cancer.aspx (accessed March 2016).

and rapidly increased, and reached a peak in 1992 following the recommendation by the American Urologic Association (AUA) and American Cancer Society (ACS) for routine prostate cancer screening with PSA. ${ }^{3}$ Likely due in part to health care organizations such as the U.S. Preventive Services Task Force (USPSTF) recommending against routine prostate cancer screening, incidence rates in the past 10 years have fallen on average $4.3 \%$ each year. ${ }^{4}$

Nationally, important epidemiologic differences in prostate cancer incidence have been discovered between different ethnicities, with African American Men (AAM) having the highest reported incidence rates. Between 2008 and 2012, AAM showed the highest yearly incidence ( 214.5 per 100,000 men), Whites and Hispanics showed intermediate incidence (130.4 and 114.7 per 100,000 men, respectively), and Asians and American Indians showing the lowest incidence (74 and 67.1 per 100,000 men, respectively) ( - Fig. 2). ${ }^{5}$ Access to care may play a central role in explaining part of these disparities, and many studies have attempted with varying results to control for socioeconomic status to evaluate the impact of this confounding variable. ${ }^{6-9}$ Biological differences have also been studied as an explanation for this significant difference in incidence among ethnicities. Overall, data suggest increased androgen axis activity in AAM to explain in part the higher incidence and higher PSA levels at the time of diagnosis seen in this ethnicity. ${ }^{10,11}$

\section{Mortality}

The majority of prostate cancer that is diagnosed among screened populations is considered low risk. While 1 man in 7 in the United States will be diagnosed with prostate cancer in his lifetime, only approximately 1 in 30 will die from the disease $e^{5} ; 98.9 \%$ of patients who were diagnosed between
2005 and 2011 survived 5 years following diagnosis. ${ }^{5}$ This overall low mortality rate is due to the relatively slowgrowing nature of many prostate cancers and the common use of PSA screening to detect early-stage disease. Mortality trends across ethnicity mimic incidence trends, with AAM faring worse in terms of cancer stage at diagnosis, as well as overall mortality. In the United States, AAM die of prostate cancer more frequently than other races/ethnicities. According to the SEER database, the overall age-adjusted prostate cancer-specific death rate was 21.4 per 100,000 men per year, with rates for AAM higher than average (46 per 100,000 men/

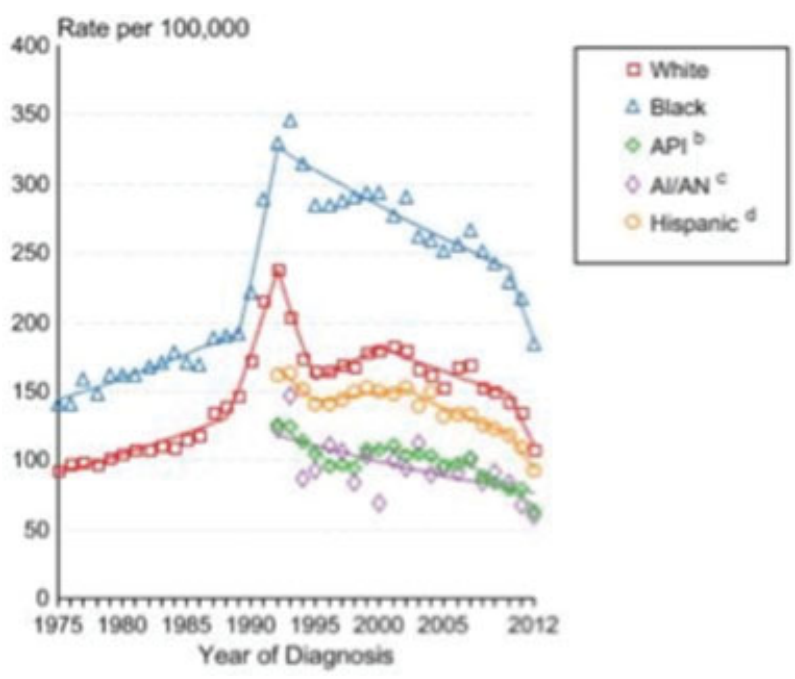

Fig. 2 SEER prostate cancer incidence from 1975 to 2012 by race/ ethnicity. Source: SEER http://seer.cancer.gov/csr/1975_2012/ browse_csr.php?sectionSEL=23\&pageSEL=sect_23_zfig.03.html (accessed March 2016). 
year); rates for Whites, American Indians, and Hispanics close to average $(20,20,18$ per 100,000 men/year, respectively); and rates for Asian Americans lower than average (9 per 100,000 men/year) (-Fig. 3).

With an estimated 307,000 deaths globally in 2012, prostate cancer is the fifth leading cause of cancer death in men ( $6.6 \%$ of total deaths) behind lung, liver, stomach, and colorectal cancer. Because PSA testing has a much greater effect on incidence than on mortality, there is less variation in mortality rates worldwide (10-fold from $\sim 3$ to 30 per $100,000)$ than is observed for incidence, with the number of deaths from prostate cancer more common in less developed than in more developed regions $(165,000$ and 142,000 , respectively) (-Fig. 3$)^{2}$

\section{Risk Factors}

Known factors that increase the risk of prostate cancer include age, family history, and race. The median age of diagnosis of prostate cancer is 66 years, with the majority of diagnoses occurring in the 65 to 74 age group. Age-adjusted incidence rates per 100,000 men sharply decline after age 75 , while mortality rates increase along with age increase. ${ }^{5}$

Prostate cancer has the highest familial cancer rate of any cancer. A single large study found a familial cancer rate of $20.2 \%$ when compared with the rates of breast $(13.6 \%)$ and colorectal cancer (12.8\%). ${ }^{12}$ The pattern of inheritance in some families is often so strong as to mimic an autosomal dominant trait. $^{13,14}$

\section{Testicular Cancer}

\section{Incidence}

When compared with prostate cancer, testicular cancer is a much less common disease, with an estimated 5.6 per 100,000 men newly diagnosed in the United States in $2012 .{ }^{15}$ Nonetheless, it is the most common cancer diagnosed in men aged 15 to 35 years. Age-specific diagnosis rates peak in men aged 25 to 29 and 30 to 43 years (14.5 and 13.7 per 100,000 men from 2008 to 2012, respectively), with lower rates in older and younger age strata. However, testicular cancer can be diagnosed at any age. Incidence varies by race, with white men having higher ageadjusted incidence rates when compared with AAM and Hispanic men (6.7 in 100,000 men compared with 1.5 in 100,000 and 4.9 in 100,000 , respectively). ${ }^{15}$ Incidence has been increasing over the past decades in the United States and other Western countries, for unknown reasons. ${ }^{16,17}$ In 2015, there were an estimated 8,430 new cases of testicular cancer diagnosed, making up $0.5 \%$ of all new cancer diagnoses in the United States. Globally, incidence rates of testicular cancer vary by geography, with higher rates in Western and Northern European nations (8.0-9.0 per 100,000) when compared with Asian and African nations ( $<1$ per 100,000), and with developed countries having higher rates than developing countries. Testicular cancer accounts for $1 \%$ of cancers in men globally. ${ }^{18}$

\section{Mortality}

The potentially curative treatment of radical orchiectomy received by these men, as well as the comparatively low burden of competing health risks in young patients, accounts in part for a high 5-year overall survival rate of $95.3 \%{ }^{15}$ The majority (68\%) of new diagnoses occur in men who present with localized disease. The 5-year survival for localized testis cancer specifically is even higher, at 99.2\%. Still, in 2015, a total of 380 men were estimated to have died from testicular cancer, accounting for $0.1 \%$ of all cancer deaths in the United States. These relatively small numbers belie the disproportionate number of life-years lost, given the young age and otherwise long life expectancy of the population most at risk

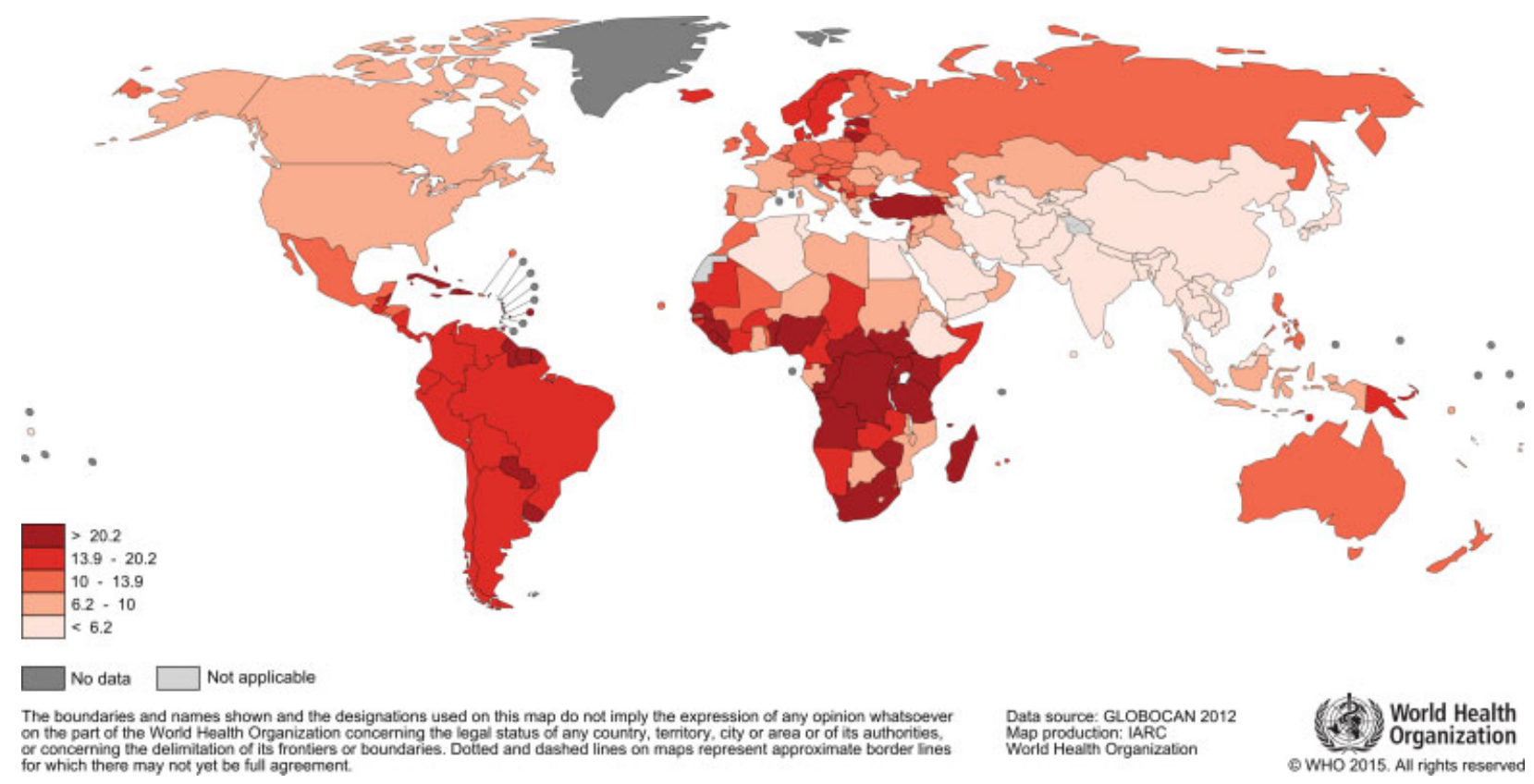

Fig. 3 Estimated prostate cancer mortality worldwide in 2012. Source: GLOBOCAN 2012 http://globocan.iarc.fr/Pages/fact_sheets_cancer.aspx (accessed March 2016). 
for testicular cancer. The death rate for testicular cancer, 0.25 per 100,000 men, has plateaued over the past two decades, speaking to the effectiveness of multimodal therapies implemented for treating this disease once it spreads beyond the testicle.

\section{Risk Factors}

Known risk factors for developing testicular cancer include cryptorchidism, a prior history of testicular cancer in the contralateral testicle, and family history. Among these factors, a prior history of the disease confers the greatest relative risk of developing testicular cancer in the contralateral testicle, with an odds ratio of 12.4 (confidence interval: $11.0-13.9) .{ }^{19}$ Other risk factors that are suspected to increase risk but continue to be under investigation include diet and environmental factors such as occupational exposures. ${ }^{20}$

\section{Summary}

Prostate and testicular cancers account for a large percentage of cancer morbidity in men in the United States and worldwide. Though patterns of incidence and mortality differ between these cancers among men of different age groups, ethnicities, and geographic locations, the overall prevalence of these cancers has been increasing over the past several decades. More men than ever are living with these men's health-specific cancers, and risk factor awareness can facilitate earlier detection and treatment.

\section{Conflict of Interest}

All authors declare no conflict of interests with this article.

\section{References}

1 Testicular Cancer. The American Cancer Society Website. Available at: http://www.cancer.org/cancer/testicularcancer/detailedguide/ testicular-cancer-key-statistics. Accessed February 15, 2016

2 Prostate cancer. GLOBOCAN 2012: International Registry for Research on Cancer Website. Available at: http://globocan.iarc. fr/Pages/fact_sheets_cancer.aspx. Accessed February 15, 2016

3 Hoffman RM, Meisner AL, Arap W, et al. Trends in United States prostate cancer incidence rates by age and stage, 1995-2012. Cancer Epidemiol Biomarkers Prev 2016;25(2):259-263
4 Zhou CK, Check DP, Lortet-Tieulent J, et al. Prostate cancer incidence in 43 populations worldwide: An analysis of time trends overall and by age group. Int J Cancer 2016;138(6):1388-1400

5 Cancer Statistics Factsheets SEER. Prostate Cancer. National Cancer Institute Web site. Available at: http://seer.cancer.gov/statfacts/ html/prost.html. Accessed February 15, 2016

6 Roetzheim RG, Pal N, Tennant C, et al. Effects of health insurance and race on early detection of cancer. J Natl Cancer Inst 1999; 91(16):1409-1415

7 Grossfeld GD, Latini DM, Downs T, Lubeck DP, Mehta SS, Carroll PR. Is ethnicity an independent predictor of prostate cancer recurrence after radical prostatectomy? J Urol 2002;168(6):2510-2515

8 Howard G, Anderson RT, Russell G, Howard VJ, Burke GL. Race, socioeconomic status, and cause-specific mortality. Ann Epidemiol 2000;10(4):214-223

9 Evans S, Metcalfe C, Ibrahim F, Persad R, Ben-Shlomo Y. Investigating Black-White differences in prostate cancer prognosis: A systematic review and meta-analysis. Int J Cancer 2008;123(2):430-435

10 Gaston KE, Kim D, Singh S, Ford OH III, Mohler JL. Racial differences in androgen receptor protein expression in men with clinically localized prostate cancer. J Urol 2003;170(3):990-993

11 Beilin J, Ball EM, Favaloro JM, Zajac JD. Effect of the androgen receptor CAG repeat polymorphism on transcriptional activity: specificity in prostate and non-prostate cell lines. J Mol Endocrinol 2000;25(1):85-96

12 Verhage BA, Aben KK, Witjes JA, Straatman H, Schalken JA, Kiemeney LA. Site-specific familial aggregation of prostate cancer. Int J Cancer 2004;109(4):611-617

13 Carter BS, Beaty TH, Steinberg GD, Childs B, Walsh PC. Mendelian inheritance of familial prostate cancer. Proc Natl Acad Sci USA 1992;89(8):3367-3371

14 Schaid DJ, McDonnell SK, Blute ML, Thibodeau SN. Evidence for autosomal dominant inheritance of prostate cancer. Am J Hum Genet 1998;62(6):1425-1438

15 Cancer Statistics Factsheets SEER. Testicular Cancer. National Cancer Institute Web site. Available at: http://seer.cancer.gov/ statfacts/html/testis.html. Accessed February 15, 2016

16 Stevenson SM, Lowrance WT. Epidemiology and diagnosis of testis cancer. Urol Clin North Am 2015;42(3):269-275

17 Le Cornet C, Lortet-Tieulent J, Forman D, et al. Testicular cancer incidence to rise by $25 \%$ by 2025 in Europe? Model-based predictions in 40 countries using population-based registry data. Eur J Cancer 2014;50(4):831-839

18 Rosen A, Jayram G, Drazer M, Eggener SE. Global trends in testicular cancer incidence and mortality. Eur Urol 2011;60(2): 374-379

19 Fosså SD, Chen J, Schonfeld SJ, et al. Risk of contralateral testicular cancer: a population-based study of 29,515 U.S. men. J Natl Cancer Inst 2005;97(14):1056-1066

20 McGlynn KA, Trabert B. Adolescent and adult risk factors for testicular cancer. Nat Rev Urol 2012;9(6):339-349 